\title{
Two novel truncating variants of the $A A A S$ gene causative of the Triple A Syndrome
}

Valeria Vezzoli $^{1 *}$, Paolo Duminuco ${ }^{1 *}$, Gabriele Pogliaghi ${ }^{1}$, Maria Saccone ${ }^{1}$, Biagio Cangiano ${ }^{1,3}$, Maria Cristina Rosatelli ${ }^{2}$, Antonella Meloni ${ }^{2}$, Luca Persani ${ }^{1,3}$, Marco Bonomi ${ }^{1,3}$

\author{
* These authors contribute equally to this work \\ era Brotzu, University of Cagliari, Cagliary, Italy; ${ }^{3}$ Department of Clinical Sciences and Community Health, \\ University of Milan, Milan, Italy;

\section{Corresponding author:} \\ Marco Bonomi, MD \\ Dipartimento di Scienze Cliniche e di Comunità - Università degli studi di Milano \\ IRCCS Istituto Auxologico Italiano - Dipartimento di Medicina Endocrino-Metabolica \\ Piazzale Brescia 20 \\ 20149 Milano \\ Phone: +39-02619112390 \\ Fax: +39-02619113033 \\ email: marco.bonomi@unimi.it
}

${ }^{1}$ IRCCS Istituto Auxologico Italiano, Division of Endocrine and Metabolic Diseases and Lab. of Endocrine and Metabolic Research, Milan, Italy; ${ }^{2}$ Pediatric Hospital "Microcitemico" Antonio Cao, Azienda Ospedali-

Short Title: $A A A S$ gene truncating variants causative of Triple A syndrome

Key words: ALADIN protein, Triple A syndrome, Congenital Hypoadrenalism, Human Splicing Finder, mRNA splicing, Allgrove syndrome

\section{Funding and Acknowledgements}

This work was supported by funds from IRCCS Istituto Auxologico Italiano (Ricerca Corrente Funds: 05C822). Authors are in debt with Professor Sungjoo Kim Yoon (Catholic University of Korea) for providing them Plasmid pEGFP-C1. 


\begin{abstract}
Purpose: The Triple-A syndrome (AAAS) is an inherited condition associated with mutations in the $A A A S$ gene, which encodes a protein of 546 amino acids known as ALADIN (ALacrima Achalasia aDrenal Insufficiency Neurologic disorder) whose function is not well understood. This protein belongs to the WD-repeat family of regulatory proteins and is located in the nuclear pore complexes. Only a few cohorts of AAAS patients have been reported and fully characterized. Thus, the objective of the present study was to report on a mini-cohort of Italian AAAS patients and to get insights on their predisposing genetic defects.

Methods: Genetic analysis of $A A A S$ gene in Triple-A syndrome patient and molecular and functional characterization of the novel identified allelic variants.

Results: Here we describe three newly diagnosed cases of AAAS, in whom genetic analysis allowed us to identify two novel allelic variants in the $A A A S$ gene: the frameshift substitution c.765 dupT (p.Gly256Trp fsX67) in exon 8 and the splice-site mutation in intron 11(c.997-2 A $>$ G, IVS11-2A $>$ G). Both variants result in a truncated non-functional protein, as we demonstrate by transcript analysis and expression studies.

Conclusions: Our findings establish a pathogenic role for both new variants. Moreover, our data highlight the essential role of the $\mathrm{C}$ terminal domain of the protein for its correct targeting and function and underline the importance of sequencing splice sites surrounding the intron-exon junctions to ensure accurate molecular diagnosis and correct genetic counseling in AAAS patients.
\end{abstract}




\section{Introduction}

Triple-A syndrome (AAAS, MIM \#231550), also known as Allgrove Syndrome, is an inherited condition characterized by the clinical triad of adrenal insufficiency, achalasia, and alacrima [1]. It is also sporadically associated with variable and progressive neurological deficiency involving the central, peripheral, and autonomic nervous systems [2]. AAAS is an autosomal recessive disorder, due to mutations in the $A A A S$ gene (Achalasia, Adrenal insufficiency, Alacrima Syndrome gene) mapping on chromosome 12q13 [3]. The pathogenic gene for Triple-A syndrome, AAAS consists of 16 exons, which encode the protein ALADIN(ALacrima Achalasia aDrenal Insufficiency Neurologic disorder) [4]. Mutations in patients with triple A syndrome are distributed through all 16 exons of the gene $[5,6]$. In addition, pathogenic mutations have been reported in introns 4, 5, 11 and 14 [7]. Mutations include point, frame-shift and nonsense mutations, as well as DNA fragment deletions. Although no specific recognition of prominent hot-spot mutations has been found, there appears to be a relatively high frequency of mutations at the following loci: Exon 1 (c.43C $>\mathrm{A}$, p. Gly14ValfsX45), exon 8 (c.787T >C, p.Ser263Pro) and intron 14 (c.1331+1G>A, IVS14+1G $>$ A) [5-8] . Noteworthy, little correlation has been found among the genotype, phenotype and variable clinical expression of family members with AAAS [6].

ALADIN is a $60-\mathrm{kDa}$ protein with a central $\approx 170$-aa domain composed of four WD repeats. The flanking $\mathrm{N}$ and $\mathrm{C}$ termini, of $\approx 150$ and 230 residues, respectively, contain no obvious structural domains. ALADIN has been shown to localize to nuclear pore complexes (NPC), a large multiprotein complex that is central for nucleocytoplasmic transport $[3,9,10]$. Accordingly, ALADIN mutations cause defects in the nuclear import of DNA repair proteins and ferritin during interphase [11-14]. Recent studies in human cells have uncovered ALADIN's role in spindle assembly via the spatial regulation of Aurora A kinase [15]. Moreover it was reported that ALADIN is essential for proper meiotic spindle assembly and positioning during meiosis in mouse oocytes, leading to severe failures in the first meiotic divisions for most oocytes [15].

There is no obvious correlation between different ALADIN mutations and disease phenotype. Patients with the same mutation, even patients within the same family, frequently display a high degree of variability in the clinical severity and age of onset of their symptoms $[16,17]$. This indicates that other factors play an im- 
portant role in the pathogenesis of AAAS.

In this study, we present a miniseries of three patients with clinical diagnosis of AAAS, who came to our attention from 2006 to 2017. All but one patient presented with an adrenal crisis. Subsequent investigation demonstrated the association with other typical features of AAAS, which led us to come to the final diagnosis, confirmed by a specific genetic analysis of the $A A A S$ gene. In particular, here we report the identification of two novel allelic variants in the ALADIN gene, the frameshift substitution c.765 dupT (p.Gly256Trp fsX67) in exon 8 and the splice-site mutation in intron 11 (c.997-2 A>G, IVS11-2A>G). For the majority of intronic variations, the consequences on mRNA splicing have been only inferred by in silico analysis, whereas here we analyzed the effect of IVS11-2A $>$ G mutation on the splicing process in silico and in vivo. Moreover, since we assessed that both mutations identified in this study lead to the formation of a truncated protein, we analyzed the expression and subcellular localization of the two novel allelic variants in a cellular environment.

\section{Materials and Methods}

\section{$\underline{\text { Mutation detection }}$}

The study, accomplishing the Declaration of Helsinki, was approved by Ethic Committee of the Istituto Auxologico Italiano, and all patients or their tutors gave a written informed consent. Genomic DNA of the probands and their parents was extracted from $3 \mathrm{~mL}$ of whole blood with Puregene DNA Purification Kit (GENTRA Systems) according to the manufacturer recommendations and stored at $-20^{\circ} \mathrm{C}$ until use. The 16 exons and the flanking intronic regions of the $A A A S$ gene (OMIM 605378) were tested for mutations by sequence analysis. PCR amplification of the $16 A A A S$ fragments was performed using a specific primer sets (available upon request). All primers were ordered from Eurofins (Ebersberg, Germany).

Each PCR product was checked on a 2\% agarose gel TAE $1 \mathrm{X}$ and purified with GFX ${ }^{\mathrm{TM}}$ PCR DNA/Gel Band Purification Kit (GE Healthcare IL, United States). the PCR product were sequenced using a Big-Dye Terminator v3.1 cycle sequencing kit (Applied Biosystem Waltham, MA, USA) according to the manufacturer's recommendations and after purification with CENTRI-SEP Spin Columns (Princeton Separations, Freehold, NJ, USA) was processed by an ABI-PRISM 3100 automated sequencer (Applied Biosystem Waltham, MA, 
USA). Sequences with DNA variations were confirmed on a different DNA aliquot.

\section{$\underline{\text { RNA Extraction and Reverse Transcriptase-Polymerase Chain Reaction (RT-PCR) }}$}

The impact of c.997-2 A>G (IVS11-2A >G) splice site mutations was assessed by RT-PCR analysis of peripheral blood leukocytes RNAs obtained from patients and parents. Total RNA was isolated from blood collected into ABI Tempus ${ }^{\mathrm{TM}}$, by using a Tempus Spin RNA Isolation Kit (Applied Biosystems) according to manufacturer instructions. Reverse transcription of total RNA was performed with SuperScript II ${ }^{\mathrm{TM}}$ Reverse Transcriptase (Invitrogen) according to the manufacturer's recommendations. Nucleic acids were quantified using the Implen Nanophotometer ${ }^{\mathrm{TM}}$ Pearl spectrophotometer. The impact of the IVS11-2A $>\mathrm{G}$ variant on the correct splicing was evaluated for patients and parents by PCR amplification, using specific primers (available upon request).

\section{$\underline{\text { Real-time quantitative PCR (qPCR) }}$}

Total RNA concentration was spectrofotometrically quantified, and structural integrity of the RNA samples was confirmed by electrophoresis in 1\% Tris-borate-EDTA (TBE)-agarose gels. After digestion with DNAse I (Sigma-Aldrich, Italy), $2 \mu \mathrm{g}$ of total RNA were retro-transcribed to cDNA via SuperScript II Reverse Transcriptase (Life Thecnologies, Italy). Real-time qPCR assays were performed in a $25 \mu 1$ reaction volume containing $12.5 \mu \mathrm{l} 2 \times$ SYBR Green I PCR Master Mix (Applied Biosystems, USA), 50 ng of cDNA, $10 \mu \mathrm{M}$ forward and reverse primers. Reactions were performed in 96-well $\mu$ ultraAmp PCR Plates-FastPlate96 (Sorenson) using the ABI Prism 7900 high-throughput sequence detection systems (Applied Biosystems) and data were processed by the associated SDS software version 2.3 (Applied Biosystems).

For each gene an amplification curve was made to evaluate the amplification efficiency. The sequences of forward and reverse primers are: AAAS_Ex8_Ex9F forward primer 5'-atgetgctatccgggtatgg-3' and AAAS_Ex12_Ex13 reverse primer 5'-cccaagcctctcctcacc-3'; GAPDH: forward primer 5'gggaagcttgtcatcaatgga-3' and reverse primer $5^{\prime}$-cgecccacttgatttgg- $3^{\prime}$. After each PCR, a melting curve was always made, to check for specificity of PCR.

\section{Construction of mutant cDNA expression vectors}


Plasmid pEGFP-C1 with full length cDNA of $A A A S$ (pEGFP-C1_AAAS_wt) was a generous gift from Professor Sungjoo Kim Yoon (Catholic University of Korea) [18][Tissue-specific expression and subcellular localization of ALADIN, the absence of which causes human triple A syndrome; [A-Ri Cho et al 2009 ]. The pEGFP-C1_AAAS_wt vector was used as template to introduce the two variations c.997-2 A>G (IVS11$2 \mathrm{~A}>\mathrm{G}$ ) and c.765dupT (p.Gly256Trp fsX67). The c.765dupT variant was performed by QuikChange SiteDirected Mutagenesis Kit ( Stratagene; La Jolla, CA, USA) according to the manufacturer's instruction using specific oligonucleotides (FW:cagttggggggcggctgctctcagcttcaccegtggatgc and REV: agccgeccccaactgggggeccaggccaagctggtaacagg) . For C.997-2 A > G variant an adapted "Two-stage PCR protocol" was used [19]. In stage one, two extension reactions were performed in separate tubes; one containing the FW1 forward primer and the other containing theREV2 reverse primer. Subsequently, the two reactions were mixed, and the standard PCR cloning procedure was carried out on the mixture. The following combination of oligonucleotides was used: Stage one-FW1 (gcttcgaattccatgtgctctctgg) + REV1 (agcagccagtctggggtcagggagcaaaaggcaggagaaggtatgtctgacagcgc) and FW2 (GCGCTGTCAGacataccttctcctgccttttgctcctgaccccagACTGGCTGCT) + REV2 (ggeccgcggttagaggtgggaat). Stage two_FW1+REV2. In both cases Pfu Turbo (Agilent) was used. Restriction enzymes used in the process were EcoRI and SacII from New England Biolabs (Beverly, MA, USA).

\section{$\underline{\text { Cells and Transfection }}$}

HeLa cells were maintained in DMEM High Glucose medium supplemented with $10 \% \mathrm{~V} / \mathrm{V}$ of fetal bovine serum (FBS) and $1 \%$ of Penicillin:Streptomycin in an incubator at $37^{\circ} \mathrm{C}$ and $5 \%$ of CO2. Twenty-four hours before transfection HeLa cells were plated at a density of $1.5 \times 10^{5}$ on sterile coverslips placed in $35 \mathrm{~mm}$ Petri dishes or on $35 \mathrm{~mm}$ Petri dishes and transfected with $2 \mu \mathrm{g}$ of the plasmid constructs using Lipofectamine ${ }^{\circledR}$ (Invitrogen) according to the manufacturer instructions. All experiments on HeLa cells were performed 2448 hours after transfection.

\section{$\underline{\text { Immunocytochemistry }}$}

Twenty-four hours after transfection, cells were washed with PBS, fixed with PBS containing 2\% paraformaldehyde (Sigma-Aldrich St. Louis, MO, USA) for $15 \mathrm{~min}$ at room temperature and rinsed twice with PBS. 
Permeabilized with $0.1 \%$ Triton in PBS for 15 minutes at RT. Blocked with $2 \%$ goat serum in PBS for 30 minutes at room temperature. Incubation happened overnight at $4^{\circ} \mathrm{C}$ with primary Anti-Nuclear Pore Complex Proteins antibody mAb414 (ab50008, ABCAM) diluted 1:200 in PBS/Goat serum. After four washes with PBS, incubation with F(ab')2-Goat anti-Mouse IgG (H+L) Cross-Adsorbed Secondary Antibody, Alexa Fluor 555 (Invitrogen) diluted 1:500 in PBS/Goat serum happened for 1h at room temperature. Cells were mounted with ProLong Gold Antifade Mountant with DAPI (Life Technologies, Carlsbad, CA, USA). Images were acquired by using a laser scanning confocal system installed on a Nikon Eclipse Ti microscope with a 60X oil immersion objective.

\section{$\underline{\text { Western blot }}$}

Twenty-four hours after transfection, HeLa cells were washed with PBS and suspended in $200 \mu$ of RIPA Buffer (10 mM Tris-HCl pH 7,2, $150 \mathrm{mM} \mathrm{NaCl}, 0,1 \%$ SDS, 1.\% Triton X-100, 1\% sodium deoxycholate, 5 mM EDTA) supplemented with protease inhibitors left 5 min at RT. Homogenates were centrifuged at 8000 rpm for 10 min at $4{ }^{\circ} \mathrm{C}$ to remove cell debris. Protein content was assayed by the BCA protein Assay Kit (Pierce, Rockford, IL 61101 USA). Total proteins were fractionated by SDS electrophoresis on NuPage 4-12 $\%$ Bis-Tris gel (Life Technologies). The iBlot ${ }^{\circledR}$ Transfer Stacks were used to transfer proteins using the iBlot ${ }^{\circledR}$ Gel Transfer Device (Invitrogen). After blocking with TBS supplemented with 5\% non-fat dry milk and $0.1 \%$ Tween 20 for 1 hour at RT, membranes were incubated overnight at $4{ }^{\circ} \mathrm{C}$ with mouse mAb antiGFP (Roche) diluted 1:2000 in TBS-T or anti-Actin (Santa Cruz Biotechnology sc-25778) diluted 1:2000 in TBS-T as internal control. After four washings in TBS-T solution 10 minutes each, membranes were incubated for $1 \mathrm{~h}$ at room temperature with a 1:4000 dilution of peroxidase-coupled goat anti-mouse antibody. Antibody-protein complexes were then detected using the Novex ECL Chemiluminescent substrate or Luminata Forte Western HRP substrate (Merck KGaA Darmstadt Germany) reagent kit (Life Technologies, Carlsbad, CA, USA) followed by autoradiography.

\section{Results}

\section{Case no 1:}

A Caucasian girl, born from unrelated unaffected parents originating from Sardinia, presented at age 3,9 
years with fatigue and hyperpigmented skin. Her physical examination was unremarkable with normal growth (height and weight at $87^{\text {th }}$ percentile; target height at $97^{\text {th }}$ percentile). The neurological examination evidenced clumsy gait. Endocrine studies confirmed adrenal insufficiency (serum cortisol=9 mcg/L, reference range 50-250 mcg/L; adrenocorticotropin, $\mathrm{ACTH}=563 \mathrm{pg} / \mathrm{ml}$, reference range 0-46 pg/mL) and normal serum aldosterone (aldosterone $14.7 \mathrm{pg} / \mathrm{ml}$, reference range 10-160 pg/ml) with normal electrolytes. Glucocorticoid replacement therapy was then promptly started (hydrocortisone, $12 \mathrm{mg}$ per day). Diagnosis of AAAS was made later at age 15, when she presented other specific features. Indeed, muscle weakness, hyperreflexia and ataxia were also noticed and the electromyoneurography demonstrating motor-sensitive polyneuropathy. Furthermore, alacrimia was diagnosed by Schirmer test. Finally, during follow-up (at the age of 18 years old), she developed feeding difficulties that led to the following diagnosis of achalasia through a specific esophagography. Moreover, at the age of 20 years the patient develops autonomic dysfunctions consisting of occasional dizziness due to orthostatic hypotension and neurogenic bladder which led to recurrent urinary tract infections (Table 1).

The genetic analysis of the $A A A S$ gene was then performed and a compound heterozygosity for previously known $A A A S$ mutations c.43C $>\mathrm{A}(\mathrm{Gly} 14 \mathrm{Valfs} X 45) / \mathrm{c} .1331+1 \mathrm{G}>\mathrm{A}(\mathrm{IVS} 14+1 \mathrm{G}>\mathrm{A})[3,5,20]$ was detected.

\section{Case no. 2}

A Caucasian boy, born from unrelated unaffected parents both with Sardinian origins, presented at 14 years because of important weight loss $(\mathrm{BMI}=14 \mathrm{~kg} / \mathrm{m} 2)$ and fatigue. At that age he has no signs of adrenarche and/or pubertal development. He had been diagnosed with achalasia one year earlier. Past history revealed congenital twisted feet and dysphagia since his 4-6 months of age. On clinical examination, cutaneousmucosal hyperpigmentation, muscle weakness and nasal speech were noted. The laboratory examination showed normal blood electrolyte as well as liver and kidney function; however, primary adrenal insufficiency was confirmed by the markedly elevated ACTH levels and low serum cortisol (serum cortisol =12 mcg/L, reference range $50-250 \mathrm{mcg} / \mathrm{L} ; \mathrm{ACTH}>1250 \mathrm{pg} / \mathrm{ml}$, reference range $0-46 \mathrm{pg} / \mathrm{mL}$ ). Serum aldosterone was normal (aldosterone $47 \mathrm{pg} / \mathrm{ml}$, reference range 10-160pg/ml). Elettromyoneurography demonstrated axonal polyneuropathy and Schirmer test was indicative for alacrimia. Glucocorticoid therapy was then immediately 
started (Table 1). Patient enters a regular pubertal development 1 year later. Genetic analysis for the $A A A S$ gene was then promptly performed. This_analysis confirmed the clinical diagnosis of AAAS revealing a novel homozygous substitution in the splice acceptor site (SAS) of intron 11 (c.997-2 A>G, IVS11-2A $>$ G) of the $A A A S$ gene. Both parents were heterozygous for the same allelic variant (Figure 1).

\section{$\underline{\text { Case no. } 3}$}

A Caucasian girl, first child of non-consanguineous unaffected parents, presented at the age of 4 years old with loss of consciousness. Clinical examination, including hormone profile, confirmed the presence of an adrenal insufficiency (serum cortisol $=10 \mathrm{mcg} / \mathrm{l}$, reference range $50-250 \mathrm{mcg} / \mathrm{L}$; ACTH $>1250 \mathrm{pg} / \mathrm{ml}$, reference range $0-46 \mathrm{pg} / \mathrm{mL}$ ). Glucocorticoid therapy was then promptly started. Two years later, at the age of 6 years old, she presented with persistent vomiting. Esophagography demonstrated the presence of megaesophagus caused by achalasia. She thus underwent to Heller myotomy followed by Nissen fundoplication. She was therefore been asked about the possible presence of combined alacrimia, that she confirmed since the birth. Schirmer test was indeed indicative for alacrimia and the final diagnosis of AAAS has been reached (Table 1). The indication for the genetic analysis was then evident and revealed a compound heterozygosity for the previously known mutation c.43C $>$ A (p.Gly14Valfs X45) in exon $1[5,20]$ and the novel frameshift mutation c.765 dupT (p.Gly256Trp fsX67) in exon 8 of $A A A S$ gene.

\section{Molecular analysis of the splicing site substitution IVS11-2A>G:}

The sequencing analysis of exon 10-intron 11 PCR product of the $A A A S$ gene of the case no.2 (Figure 2) revealed the splicing site mutation c.997-2 A $>$ G, IVS11-2A $>$ G in the homozygous state. This variation touches the splicing acceptor site of exon 11 and has not been previously reported in the scientific literature. The Human Splicing Finder (HSF) prediction software [21] predicted that the wild-type acceptor site was modified suggesting the abolition of the broken wild-type site and the creation of a new cryptic splicing acceptor site (Table 2) with the final insertion of a 36 bp intronic sequence. Indeed RT-PCR analysis performed on blood samples showed a single band in the normal subject at the expected size of $382 \mathrm{bp}$, whereas a higher band was present in the proband (Figure 2). The purification and sequencing of the cDNA band of the patient confirm HSF prediction with the insertion of an intronic sequence of $36 \mathrm{bp}$ which determine the introduction 
of 9 unrelated amino acids and a premature termination codon (PTC) after exon 10 (Figure 2).

Since this mutation at the splicing acceptor site is expected to generate a truncated protein, we hypothesize that the nonsense-mediated mRNA decay (NMD) is triggered. This prompted us to analyze NMD in proband and heterozygous carriers (parents), measuring the expression levels of ALADIN transcript. Our findings revealed a highly reduced expression of the mutated allele as an expected result of the activation of the NMD process (Figure 3A). Though the expression of the mutated allele was very low and the mutation was predicted to generate a truncated protein, the mutated and non-mutated molecules shared the first 332 amino acids, including four WD repeat responsible of the assembly of macromolecular complexes [22]. This made us wonder if the mutated protein could conserve in part the ability to localize to NPCs, where it could exert partially its function. The same consideration must be done also for the novel frameshift mutation in exon 8 (c.765 dupT- p.Gly256Trp fsX67) that leads to a truncated protein partially preserving (until amino acid 255) the $\mathrm{N}$ terminal portion of ALADIN protein.

\section{Subcellular localization of naturally occurring truncating mutations}

The C-terminal portion of ALADIN was reported to be crucial for the function of the protein itself $[3,9,16$, 17, 23-25], indeed numerous mutations identified in AAAS patients result in truncation of this domain. Thus, we analyzed the expression and subcellular localization of the two novel allelic variants IVS11-2A>G and c.765 dupT identified in our patients; since both mutations resulted in a protein deprived of the Cterminal domain. Even if the two mutant proteins are expressed in Hela cells (Figure 3B), we observe qualitative alterations in their subcellular localization.

After transfection of GFP-ALADIN and mutant vectors, the GFP-ALADINR332fs (c.997-2 A $>$ G) no longer localizes to NPCs but is found predominantly in the cytoplasm (Figure 4B; compare with wild-type localization in Figure 4A). Similar results were obtained also with the GFP-ALADING256fs (c.765 dupT) mutant (Figure 4C).

\section{Discussion}

AAAS is a rare autosomal recessive congenital disease characterized by adrenal insufficiency (Addison's disease), failure of the lower esophageal sphincter to relax (achalasia) and an absence of tear secretion (alac- 
rima). In addition to the three specific features (achalasia, Addison's disease, and alacrimia), AAAS patients can develop muscle weakness and neurological abnormalities consisting of autonomic, sensory and motor neuropathies. The neurological manifestations has more commonly been reported in older individuals, but they can be detected at an early age [26] and can worsen over time.

The AAAS diagnosis is difficult because of its rarity (the prevalence of disease is unknown) and clinical onset heterogeneity. Being AAAS a multisystem and progressive disease, the diagnosis could be delayed, due to the long latency observed between the first and the others known signs of the pathognomonic triad [27]. Consistently, in the present study we report three novel patients whose final diagnosis of AAAS was achieved after a variable period from the first clinical manifestation. Notably, in patient no.1 and no.2, latency between the earliest clinical feature and final diagnosis of AAAS was more than10 years (Table1). In particular, while patient 2 showed axonal polyneuropathy at 14 years old and patient 3 is so far free of symptoms, patients 1 showed first neurological signs already at diagnosis (age 3.9 years) with their worsening in the young adulthood (age 20 years). It is worth to underline that the regular follow up of this patient allowed the early diagnosis and correct management of new manifestations. Thus, our data in according with literature suggest that neurological abnormalities should be controlled life-long in patients with a confirmed genetic cause.

Genetic analysis confirmed AAAS diagnosis revealing the presence of two previously described (c.43C $>$ A(p.Gln15LyS)/ c.1331+1G $>$ A (IVS14+1G $>$ A) mutations in exon 1 and intron 14 and, most importantly, two novel variants in the $A A A S$ gene, resulting in truncated non-functional proteins (Figure 1). Two of our cases (no.1 and no.3) were presenting a partial genotype identity (c. 43C >A, p.Gly14ValfsX45) but a quite different phenotype presentation/progression and complexity with a more acute and severe of Addison's disease presentation in case no. 3 (adrenal crisis with loss of consciousness); on the other hand the neurological degenerative features, found in patient no. 1, are the main prognostic factor in AAAS patients, once the hormonal defect has been replaced $[7,8]$. These differences might be related to the second heterozygous allelic variant but could also confirm the known absence of genotype-phenotype correlation for the 
$A A A S$ gene allelic variants [9]. No one of our patients showed a mineralocorticoid defect, described in $15 \%$ of AAAS patients [28, 29].

The c.765 dupT (p.Gly256Trp fsX67) in exon 8, a region that has been associated with a high frequency of mutations, is a frameshift mutation not reported previously. This mutation results in the substitution of Glycine at amino acid position 256 by Tryptophan leading to the insertion of 67 amino acids alternative to Aladin sequence and followed by a premature termination codon (PTC). Further evaluation revealed that this truncated mutant no longer localizes to NPCs but is found predominantly in the cytoplasm (Figure 4B), likely associated with the loss of nucleocytoplasmic transport activity.

The novel splice site mutation c.997-2 A>G (IVS11-2A $>$ G) turns the A at position c.997-2 into G, losing the consensus splice acceptor site (SAS) but creating a new (SAS) upstream in the intron 10, as in silico analysis predicted and mRNA analysis demonstrated (Figure 2). The use of the novel splicing site leads to the insertion of an intronic sequence of $36 \mathrm{bp}$ which cause the introduction of 9 amino acids non related with ALADIN protein followed by a PTC after exon 10. This mutation at the SAS was predicted to trigger the nonsense-mediated mRNA decay (NMD). NMD is a surveillance pathway, whose main function is to reduce errors in gene expression by abolishing mRNA transcripts that contain premature termination codons. This prevents the translation of aberrant transcripts and limits the synthesis of abnormal proteins [30], such as the transcripts generated from the mutated allele. This prompted us to analyze NMD in proband and heterozygous carrier (father), determining the expression levels of ALADIN transcript. Our findings revealed a significant reduction of the mutated allele expression as an expected result of the activation of the NMD process (Figure 3A). Even if the expression of the mutated allele was low and the mutation at the (SAS) sequence was predicted to generate a truncated protein, the mutated and non-mutated proteins shared the first 332 amino acids, including four WD repeat responsible of the assembly of macromolecular complexes [22]. Thus, we analyzed the expression and subcellular localization of this novel allelic variant (IVS11-2A $>$ G). Even if the GFP-ALADIN mutant expression is partially conserved after overexpression in Hela cells (Figure 3B), we observe qualitative differences in its subcellular localization. In particular we observe that the mutated protein is found predominantly in the cytoplasm. Similar results were obtained with several other pathogenic 
ALADIN mutations that disrupt larger stretches of the $\mathrm{C}$ terminus. Almost all known nonsense, frameshift, and splice-site mutations that encode for an ALADIN protein lacking this domain will not localize to NPCs. Our findings confirm that the C-terminal domain of ALADIN is essential for its correct targeting to NPCs (Figure 4). Besides, the observation that this domain is deleted in a large number of AAAS patients point out that NPC targeting is essential for normal ALADIN function [20, 31].

In conclusion, we report two novel variants in the $A A A S$ gene, both resulting in a truncated non-functional ALADIN protein. No clear-cut genotype-phenotype correlation could be deduced from our study, a lack of which has been seen in other studies as well [32][33]. Nonetheless, our findings highlight the essential role of the $\mathrm{C}$ terminal domain of the protein for its correct targeting and function and underline the importance of sequencing splice sites surrounding the intron-exon junctions and the need to carry out additional RNA studies to ensure precise molecular diagnosis and correct genetic counseling.

\section{Conflict of interest:}

The authors declare that they have no conflict of interest.

\section{References:}

1. Allgrove J, Clayden GS, Grant DB, Macaulay JC (1978) Familial glucocorticoid deficiency with achalasia of the cardia and deficient tear production. Lancet (London, England) 1:1284-6

2. Huebner A, Elias LL, Clark AJ (1999) ACTH resistance syndromes. J Pediatr Endocrinol Metab 12 Suppl 1:277-93

3. Tullio-Pelet A, Salomon R, Hadj-Rabia S, et al (2000) Mutant WD-repeat protein in triple-A syndrome. Nat Genet 26:332-335. https://doi.org/10.1038/81642

4. Weber A, Wienker TF, Jung M, et al (1996) Linkage of the gene for the triple A syndrome to chromosome 12q13 near the type II keratin gene cluster. Hum Mol Genet 5:2061-6. https://doi.org/10.1093/hmg/5.12.2061

5. Papageorgiou L, Mimidis K, Katsani KR, Fakis G (2013) The genetic basis of triple A (Allgrove) 
syndrome in a Greek family. Gene 512:505-509. https://doi.org/10.1016/j.gene.2012.10.008

6. Huebner A, Kaindl AM, Knobeloch KP, et al (2004) The triple A syndrome is due to mutations in ALADIN, a novel member of the nuclear pore complex. Endocr Res 30:891-9

7. Vallet A-E, Verschueren A, Petiot P, et al (2012) Neurological features in adult Triple-A (Allgrove) syndrome. J Neurol 259:39-46. https://doi.org/10.1007/s00415-011-6115-9

8. Roucher-Boulez F, Brac de la Perriere A, Jacquez A, et al (2018) Triple-A syndrome: a wide spectrum of adrenal dysfunction. Eur J Endocrinol 178:199-207. https://doi.org/10.1530/EJE-170642

9. Handschug K, Sperling S, Yoon SJ, et al (2001) Triple A syndrome is caused by mutations in AAAS, a new WD-repeat protein gene. Hum Mol Genet 10:283-90. https://doi.org/10.1093/hmg/10.3.283

10. Cronshaw JM, Krutchinsky AN, Zhang W, et al (2002) Proteomic analysis of the mammalian nuclear pore complex. J Cell Biol 158:915-27. https://doi.org/10.1083/jcb.200206106

11. Hirano M, Furiya Y, Asai H, et al (2006) ALADINI482S causes selective failure of nuclear protein import and hypersensitivity to oxidative stress in triple A syndrome. Proc Natl Acad Sci U S A 103:2298-303. https://doi.org/10.1073/pnas.0505598103

12. Storr HL, Kind B, Parfitt DA, et al (2009) Deficiency of ferritin heavy-chain nuclear import in triple a syndrome implies nuclear oxidative damage as the primary disease mechanism. Mol Endocrinol 23:2086-94. https://doi.org/10.1210/me.2009-0056

13. Prasad R, Metherell LA, Clark AJ, Storr HL (2013) Deficiency of ALADIN impairs redox homeostasis in human adrenal cells and inhibits steroidogenesis. Endocrinology 154:3209-18. https://doi.org/10.1210/en.2013-1241

14. Jühlen R, Idkowiak J, Taylor AE, et al (2015) Role of ALADIN in human adrenocortical cells for oxidative stress response and steroidogenesis. PLoS One 10:e124582. https://doi.org/10.1371/journal.pone.0124582

15. Carvalhal S, Ribeiro SA, Arocena M, et al (2015) The nucleoporin ALADIN regulates Aurora A localization to ensure robust mitotic spindle formation. Mol Biol Cell 26:3424-3438. 
https://doi.org/10.1091/mbc.E15-02-0113

16. Sandrini F, Farmakidis C, Kirschner LS, et al (2001) Spectrum of mutations of the AAAS gene in Allgrove syndrome: lack of mutations in six kindreds with isolated resistance to corticotropin. J Clin Endocrinol Metab 86:5433-7. https://doi.org/10.1210/jcem.86.11.8037

17. Goizet C, Catargi B, Tison F, et al (2002) Progressive bulbospinal amyotrophy in Triple A syndrome with AAAS gene mutation. Neurology 58:962-965. https://doi.org/10.1212/WNL.58.6.962

18. Cho AR, Yang KJ, Bae Y, et al (2009) Tissue-specific expression and subcellular localization of ALADIN, the absence of which causes human triple A syndrome. Exp Mol Med 41:381-6. https://doi.org/10.3858/emm.2009.41.6.043

19. Wang W, Malcolm BA (1999) Two-stage PCR protocol allowing introduction of multiple mutations, deletions and insertions using QuikChange(TM) Site-Directed Mutagenesis. Biotechniques 26:680682. https://doi.org/10.2144/99264st03

20. Krumbholz M, Koehler K, Huebner A (2006) Cellular localization of 17 natural mutant variants of ALADIN protein in triple A syndrome - shedding light on an unexpected splice mutation. Biochem Cell Biol 84:243-9. https://doi.org/10.1139/o05-198

21. Desmet F-O, Hamroun D, Lalande M, et al (2009) Human Splicing Finder: an online bioinformatics tool to predict splicing signals. Nucleic Acids Res 37:e67. https://doi.org/10.1093/nar/gkp215

22. Smith TF, Gaitatzes C, Saxena K, Neer EJ (1999) The WD repeat: a common architecture for diverse functions. Trends Biochem Sci 24:181-5

23. Persic M, Prpić I, Huebner A, Severinski S (2001) Achalasia, alacrima, adrenal insufficiency, and autonomic dysfunction: double A, triple A, or quaternary A syndrome? J Pediatr Gastroenterol Nutr $33: 503-4$

24. Schmittmann-Ohters K, Huebner A, Richter-Unruh A, Hauffa BP (2001) Clinical and novel molecular findings in a 6.8-year-old Turkish boy with triple A syndrome. Horm Res 56:67-72. https://doi.org/10.1159/000048093

25. Houlden H, Smith S, De Carvalho M, et al (2002) Clinical and genetic characterization of families 
with triple A (Allgrove) syndrome. Brain 125:2681-90. https://doi.org/10.1093/brain/awf270

26. Gazarian M, Cowell CT, Bonney M, Grigor WG (1995) The "4A" syndrome: adrenocortical insufficiency associated with achalasia, alacrima, autonomic and other neurological abnormalities. Eur J Pediatr 154:18-23. https://doi.org/10.1007/bf01972967

27. Patt H, Koehler K, Lodha S, et al (2017) Phenotype-genotype spectrum of AAA syndrome from Western India and systematic review of literature. Endocr Connect 6:901-913. https://doi.org/10.1530/EC-17-0255

28. Clark AJL, Weber A (1998) Adrenocorticotropin Insensitivity Syndromes. Endocr Rev 19:828-843. https://doi.org/10.1210/edrv.19.6.0351

29. Collares CVA, Antunes-Rodrigues J, Moreira AC, et al (2008) Heterogeneity in the molecular basis of ACTH resistance syndrome. Eur J Endocrinol 159:61-8. https://doi.org/10.1530/EJE-08-0079

30. Maquat LE (2004) Nonsense-mediated mRNA decay: splicing, translation and mRNP dynamics. Nat Rev Mol Cell Biol 5:89-99. https://doi.org/10.1038/nrm1310

31. Cronshaw JM, Matunis MJ (2003) The nuclear pore complex protein ALADIN is mislocalized in triple A syndrome. Proc Natl Acad Sci U S A 100:5823-7. https://doi.org/10.1073/pnas.1031047100

32. Dumic M, Barišic N, Kusec V, et al (2012) Long-term clinical follow-up and molecular genetic findings in eight patients with triple A syndrome. Eur J Pediatr 171:1453-1459. https://doi.org/10.1007/s00431-012-1745-1

33. Prpic I, Huebner A, Persic M, et al (2003) Triple A syndrome: genotype-phenotype assessment. Clin Genet 63:415-7 


\section{Figure Legend}

Figure 1. Family pedigree and detection of the c.992-2A $>G$ and $c .765 d u p ~ T$ mutations

Pedigree of the two families. Direct sequence analysis of the $A A A S$ gene identified the two novel mutations of $A A A S$ gene c.997-2A $>\mathrm{G}$ and c.765dup $\mathrm{T}$.

\section{Figure 2. Sequencing representation of the cDNA analysis}

(A) Gel electrophoresis of the RT-PCR amplification. MW = 50bp DNA Step Ladder ( Promega); WT = normal; $\mathrm{P}=$ patient; (B) chromatogram and schematic representation of the mutated allele, including exon 10, part of intron 10 and exon 11. The RED BOX indicates the STOP GAIN.

Figure 3. Nonsense pre-mRNA mediated decay analysis. (A) mRNA expression of AAAS WT (CTRL) and mutated allele (PROBAND) by qPCR performed on blood samples. GAPDH mRNA was used to normalize template levels. Values are mean \pm SEM and represent the relative expression compared with controls (AAAS/GAPDH mRNA levels). qPCR experiments were performed in triplicate wells for each subject and repeated on two different aliquot of blood . (B) Western blot analysis of Hela cells expressing the wild-type GFP-ALADIN (1) the GFP-ALADINR332fs (c.997-2 A>G) (2) variant or GFP-ALADING256fs (c.765 dupT) (3) variant. Cells transfected with the empty vector (GFP alone) were loaded as control (4). While in the GFP-ALADIN an accumulation of a main band with molecular masses of 90-100 kDa was observed, in the mutated proteins we observed a lower molecular mass signal, as expected for truncated proteins. Antiactin antibody was used as internal loading control. The presence of higher molecular weight faint bands for WT and mutated proteins is possibly due to the GFP tag, as higher bands $(>27 \mathrm{kDa})$ were present also in the cells transfected with GFP protein only.

Fig. 4. The C-terminal domain of ALADIN is essential for NPC targeting. The localization of GFPtagged ALADIN constructs is shown on the right of each image (green). (A) Wild-type ALADIN. (B) ALADING256fs variant (C) the ALADINR332fs variant. The localization of NPCs is shown on the middle of each image (red) by immunostaining with mAb414 (recognizes Nup358, Nup214, Nup153, and Nup62). The merged signal is shown on the left of each image. 
Table 1. Clinical summary of the patients.

\begin{tabular}{|c|c|c|c|c|c|c|c|c|c|c|c|c|c|c|}
\hline Patient & Sex & $\begin{array}{l}\text { Age } \\
\text { at } \\
\text { onset } \\
\text { (yr) }\end{array}$ & $\begin{array}{l}\text { Complaints } \\
\text { on } 1^{\text {st }} \\
\text { admission }\end{array}$ & $\begin{array}{c}\text { Age at } \\
\text { definitive } \\
\text { TAS } \\
\text { diagnosis } \\
\text { (year) }\end{array}$ & $\begin{array}{c}\mathrm{AI} \\
(\mathbf{y r})^{a}\end{array}$ & $\begin{array}{c}\mathrm{AL} \\
(\mathbf{y r})^{a}\end{array}$ & $\begin{array}{c}\mathrm{AC} \\
(\mathbf{y r})^{a}\end{array}$ & $\begin{array}{r}\text { HR } \\
(\mathbf{y r})^{a}\end{array}$ & $\begin{array}{l}\text { MW } \\
(\mathbf{y r})^{a}\end{array}$ & $\begin{array}{l}\text { NS } \\
(\mathbf{y r})^{a}\end{array}$ & $\begin{array}{c}\mathrm{AT} \\
(\mathbf{y r})^{a}\end{array}$ & $\begin{array}{c}\text { PN } \\
(\mathbf{y r})^{a}\end{array}$ & $\begin{array}{c}\mathbf{A N} \\
(\mathbf{y r})^{a}\end{array}$ & $\begin{array}{c}A A A S \text { gene allelic } \\
\text { variant }\end{array}$ \\
\hline 1 & $\mathrm{~F}$ & 3.9 & $\begin{array}{l}\text { fatigue and } \\
\text { hyper- } \\
\text { pigmented } \\
\text { skin }\end{array}$ & 15 & $\begin{array}{c}+ \\
(3.9)\end{array}$ & $\begin{array}{c}+ \\
(15)\end{array}$ & $\begin{array}{c}+ \\
(18)\end{array}$ & $\begin{array}{c}+ \\
(15)\end{array}$ & $\begin{array}{c}+ \\
(15)\end{array}$ & $\begin{array}{c}+ \\
(12)\end{array}$ & $\begin{array}{c}+ \\
(15)\end{array}$ & $\begin{array}{c}+ \\
(15)\end{array}$ & $\begin{array}{c}+ \\
(20)\end{array}$ & $\begin{array}{c}\text { c. } 43 \mathrm{C}>\mathrm{A} \\
\text { (p.Gly14ValfsX45) } \\
\text { c.1331+1G }>A \\
(\text { IVS } 14+1 \mathrm{G}>\mathrm{A}) \\
\end{array}$ \\
\hline 2 & $\mathrm{M}$ & 0.6 & $\begin{array}{l}\text { weight loss } \\
\text { and fatigue }\end{array}$ & 14 & $\begin{array}{c}+ \\
(14)\end{array}$ & $\begin{array}{c}+ \\
(14)\end{array}$ & $\begin{array}{c}+ \\
(0.6)\end{array}$ & $\begin{array}{c}+ \\
(14)\end{array}$ & $\begin{array}{c}+ \\
(14)\end{array}$ & $\begin{array}{c}+ \\
(14)\end{array}$ & $\begin{array}{c}+ \\
(14)\end{array}$ & $\begin{array}{c}+ \\
(14)\end{array}$ & - & $\begin{array}{l}\text { c. } 997-2 \mathrm{~A}>\mathrm{G} \\
(\mathrm{IVS} 11-2 \mathrm{~A}>\mathrm{G})\end{array}$ \\
\hline 3 & $\mathrm{~F}$ & 4 & $\begin{array}{c}\text { loss of } \\
\text { consciousness }\end{array}$ & 6 & $\begin{array}{c}+ \\
(4)\end{array}$ & $\begin{array}{l}+ \\
(6)\end{array}$ & $\begin{array}{l}+ \\
(6)\end{array}$ & $\begin{array}{l}+ \\
(6)\end{array}$ & $\begin{array}{l}+ \\
(6)\end{array}$ & $\begin{array}{l}+ \\
(6)\end{array}$ & $\begin{array}{c}+ \\
(6)\end{array}$ & - & - & $\begin{array}{c}\text { c.43C }>\text { A } \\
\text { (p.Gly14ValfsX45) } \\
\text { c.765 dupT } \\
\text { (p.Gly256TrpfsX67) }\end{array}$ \\
\hline
\end{tabular}

Legend: $\mathrm{AI}=$ adrenal insufficiency; $\mathrm{AL}=$ alacrimia; $\mathrm{AC}$ : achalasia/swallowing difficulties; $\mathrm{HR}=$ hyperreflexia; MW: muscle weakness; NS= nasal speech; AT= ataxia/clumsiness; $\mathrm{PN}=$ polyneuropathy; $\mathrm{AN}=$ autonomic neuropathy; ${ }^{a}$ Number in parentheses indicates age when the AAAS symptoms appeared. 
Table 2. HSF prediction of the c.997-2A>G mutation on the splicing (http://www.umd.be/HSF3/index.html)

\begin{tabular}{|c|c|c|c|c|c|c|}
\hline $\begin{array}{c}\text { cDNA } \\
\text { Position }\end{array}$ & $\begin{array}{c}\text { Splice } \\
\text { Site type }\end{array}$ & Motif & $\begin{array}{c}\text { New splice } \\
\text { site }\end{array}$ & $\begin{array}{c}\text { Wild } \\
\text { Type }\end{array}$ & Mutant & $\begin{array}{c}\text { Variation } \\
\text { (\%) }\end{array}$ \\
\hline c.997-12 & Acceptor & ccctgaccccagAC & ccctgaccceggAC & 86.21 & 57.26 & $\begin{array}{c}\text { WT site } \\
\text { broken } \\
-33.58\end{array}$ \\
\hline c.997-48 & Acceptor & attctactatagac & attctactatagAC & 80.07 & 80.07 & New splicing site \\
\hline
\end{tabular}


A

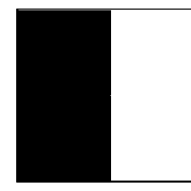
c.997-2A $>G$

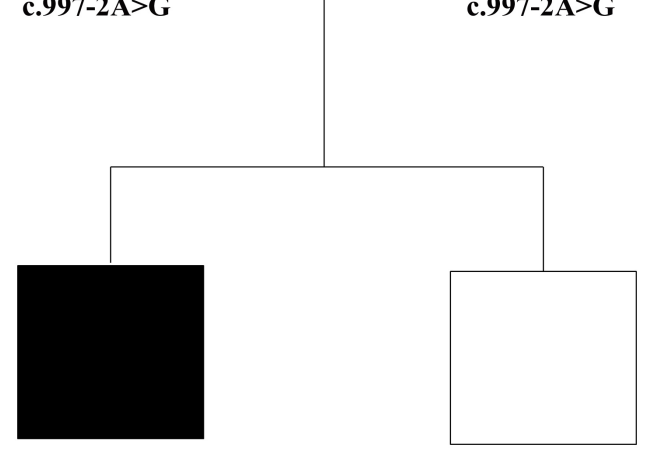

c. $997-2 \mathrm{~A}>\mathrm{G}$ c.997-2A $>G$

B
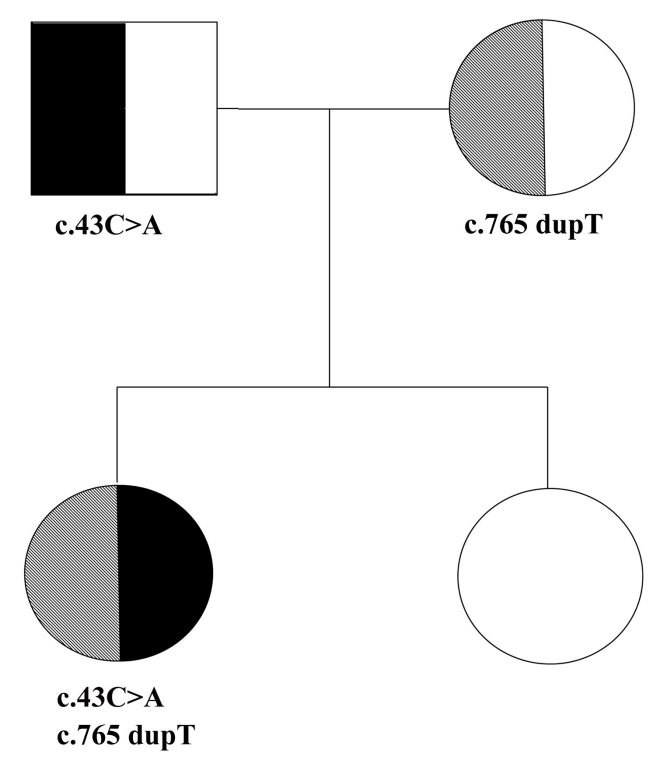
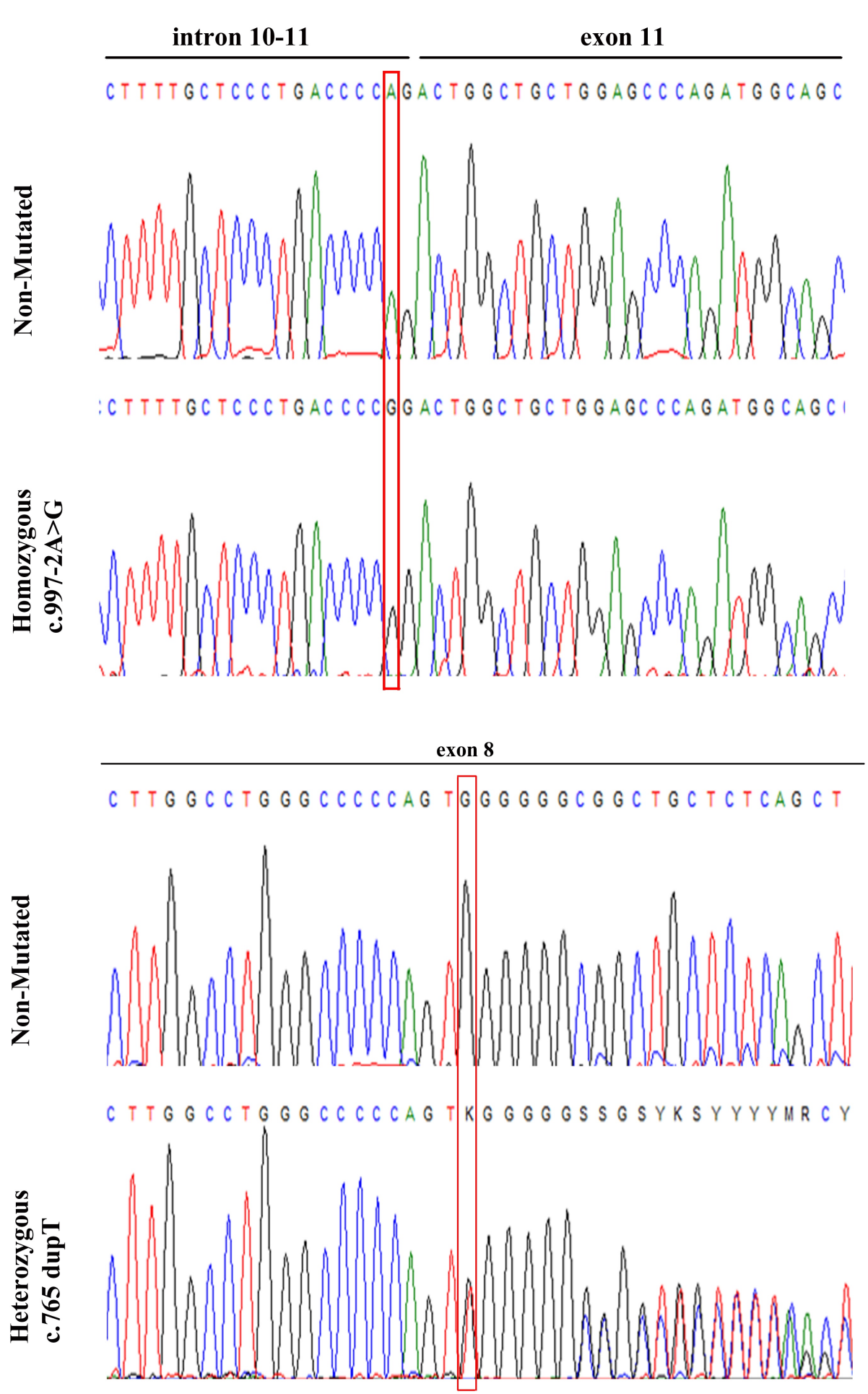
A

MW WT P

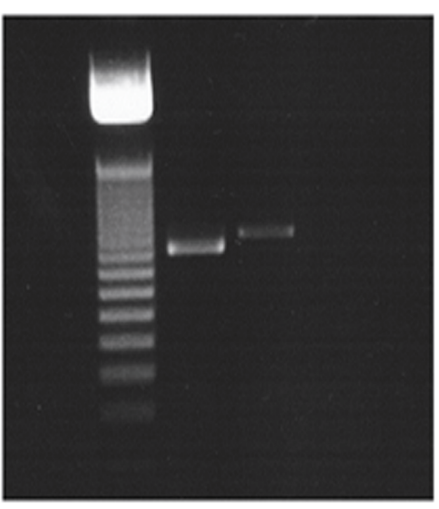

B
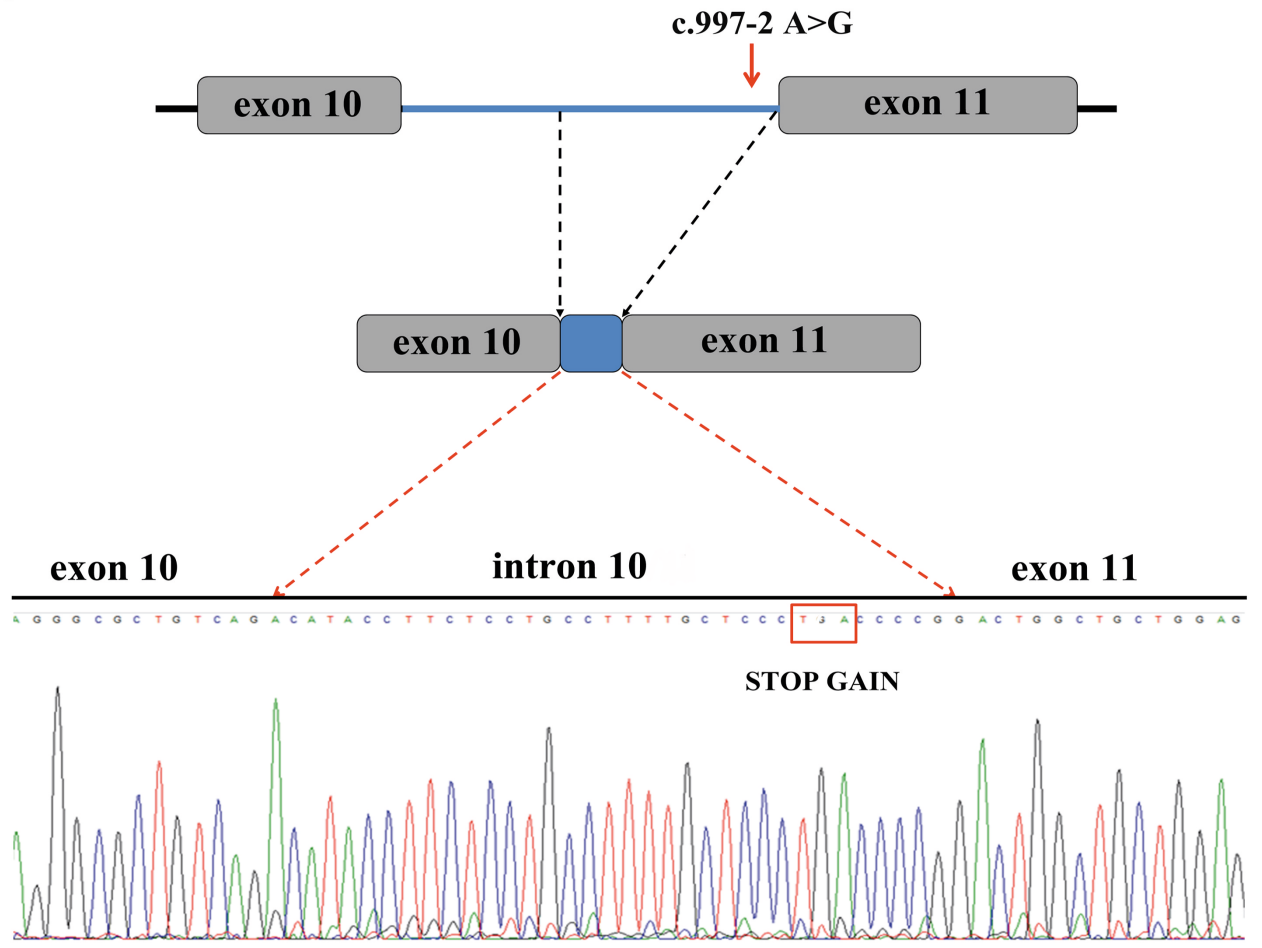
A

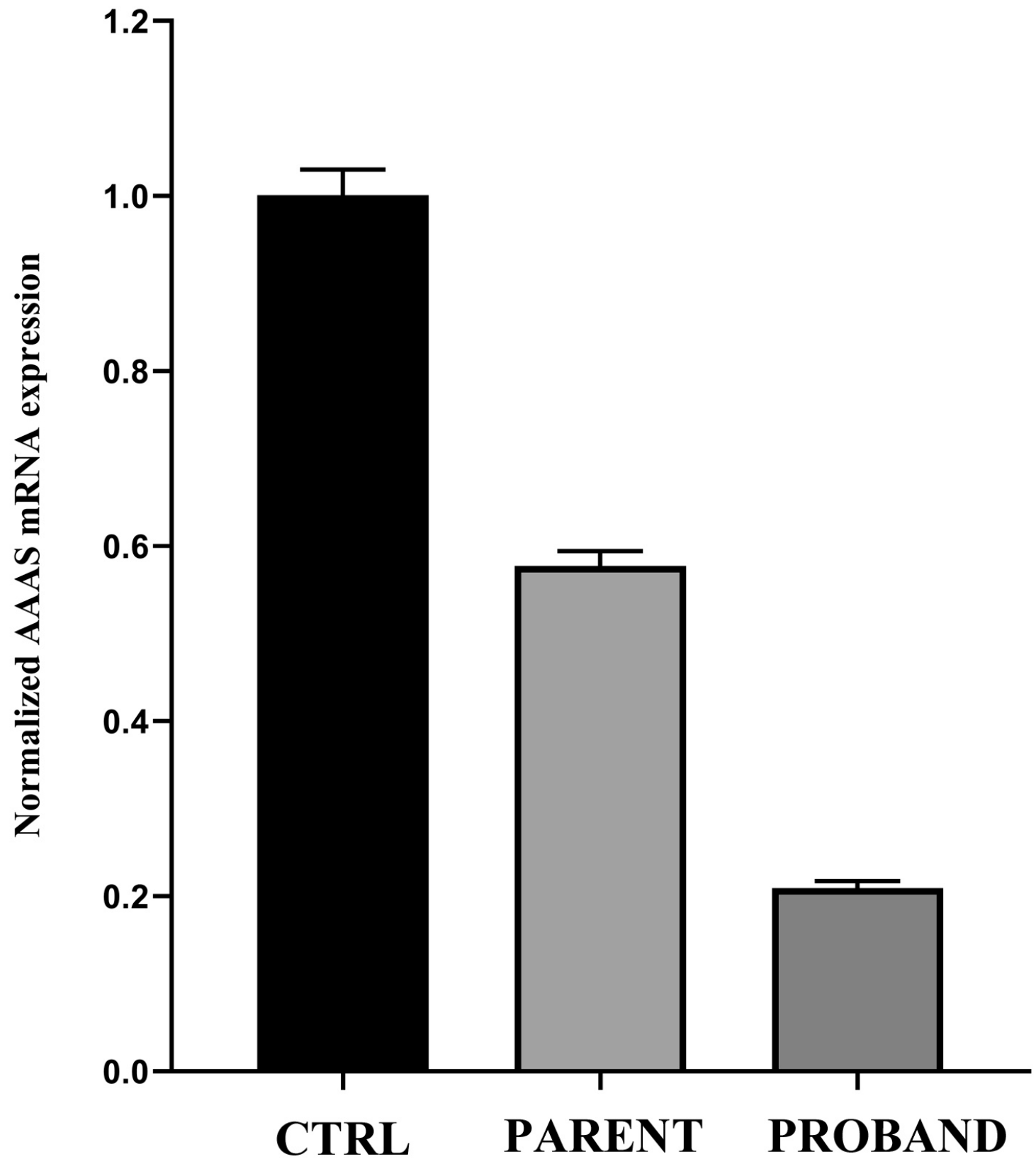

B

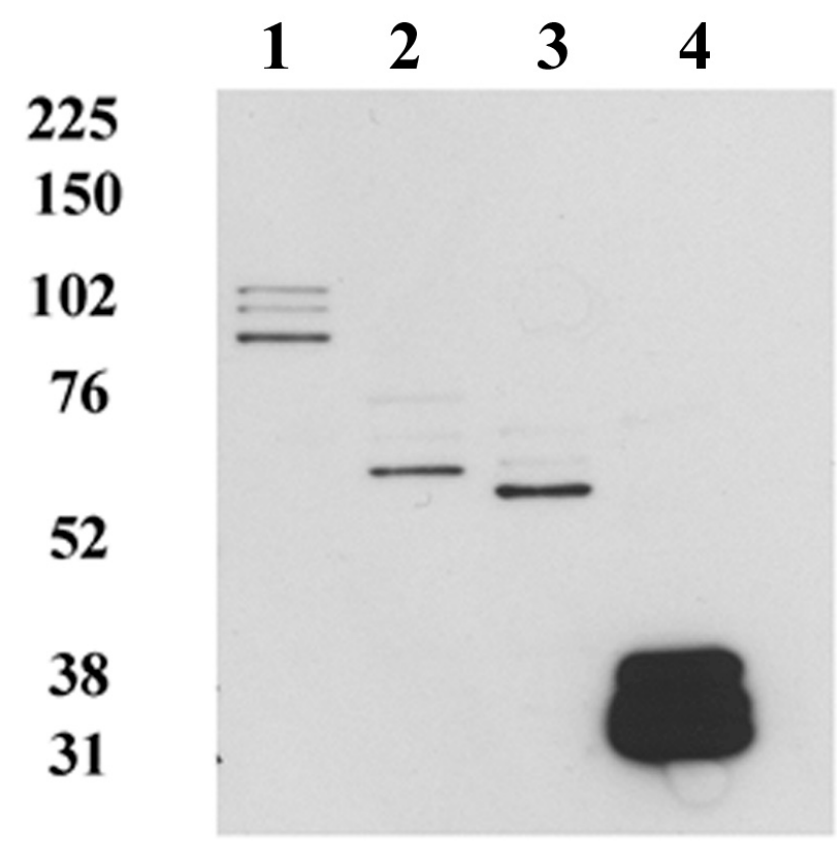




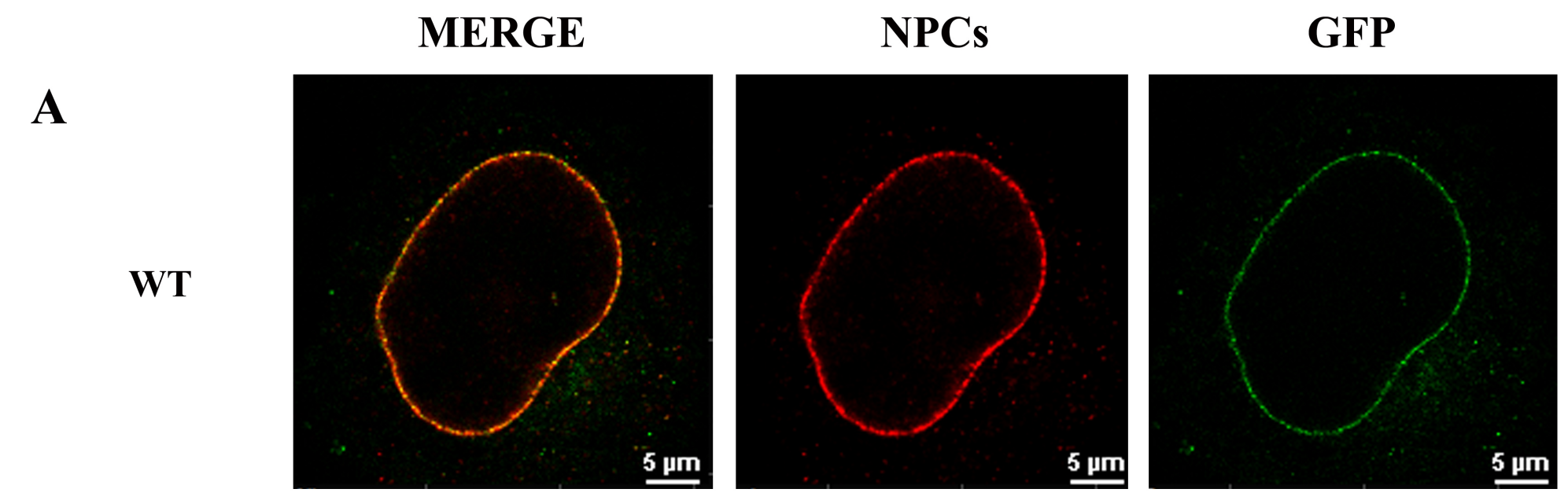

B

ALADING256fs
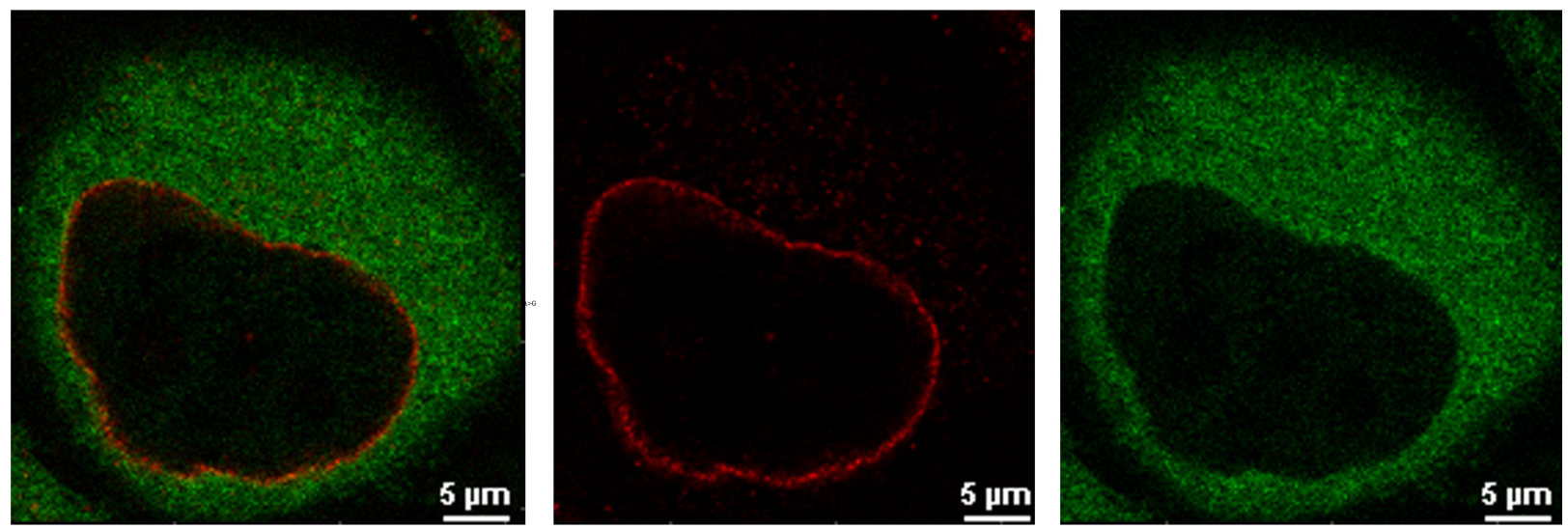

C

ALADINR332fs
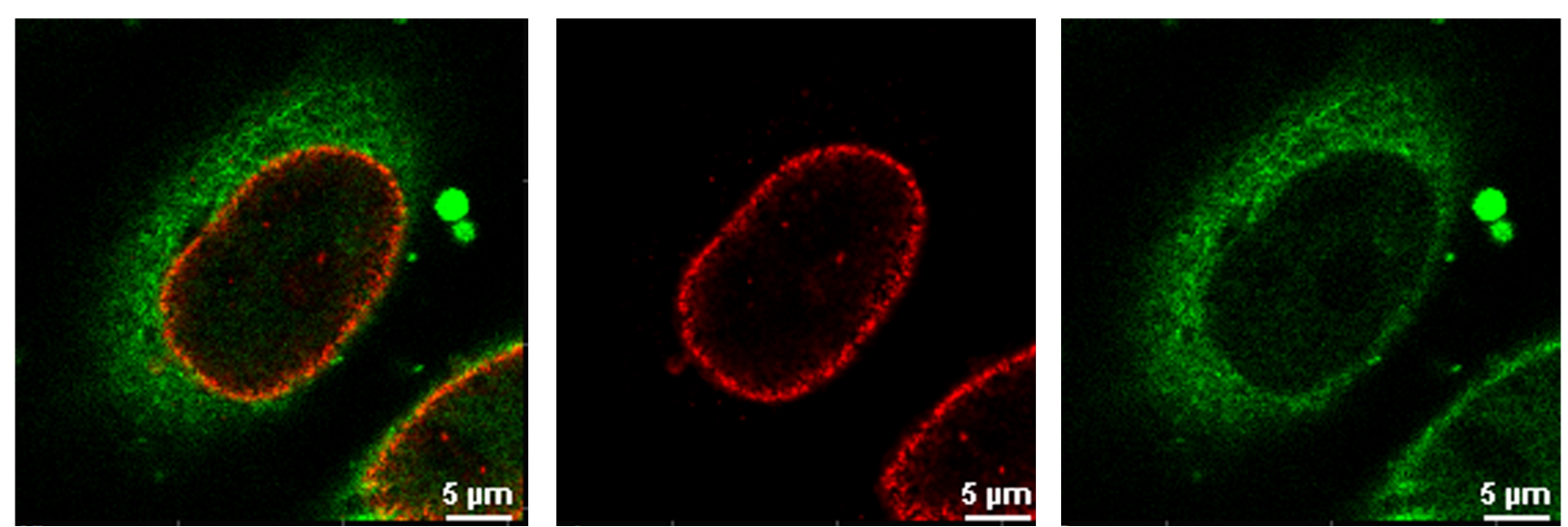